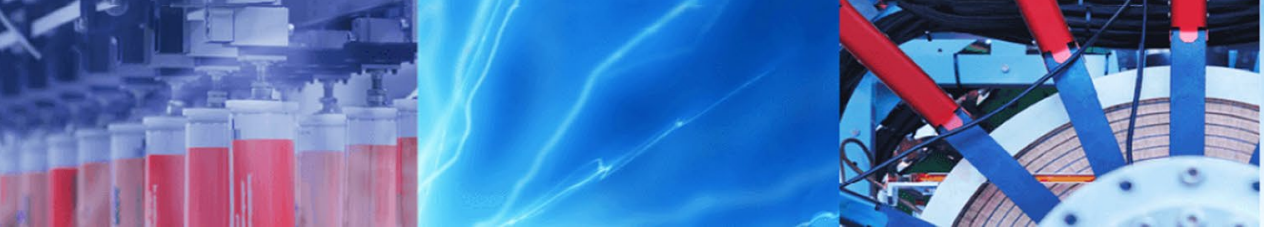

Research Article

\title{
Preparation and evaluation of amine terminated polyether shale inhibitor for water-based drilling fluid
}

\author{
Xiaodong Bai ${ }^{1}$ Xingyuan Zhang ${ }^{1} \cdot$ Vasileios Koutsos $^{2} \cdot$ Zhihong Fu $^{1} \cdot$ Tao Ning $^{1} \cdot$ Yumei Luo ${ }^{1} \cdot$ Song Zhou ${ }^{1}$
}

(c) Springer Nature Switzerland AG 2018

\begin{abstract}
Facing the problems of shale hydration, swelling and borehole wall instability in shale gas drilling, a new type of amine terminated polyether (ATPE) shale inhibitor used in water-based drilling fluid is successfully synthesized through the method of leaving group. Using the yield of ATPE as index, orthogonal experiments are designed to determine the optimal reaction conditions. When the mole ratio of polyethylene glycol tosylate (PEG-OTs)/ethylenediamine is $1 / 3.6$, mole ratio of PEG-OTs/triethylamine (acid binding agent) is $1 / 4$, reaction time is $3.5 \mathrm{~h}$ and reaction temperature is $60^{\circ} \mathrm{C}$, the yield of ATPE is $91.12 \%$. The structure and molecular weight of polyethylene glycol (PEG-400, a kind of oligomer), PEG-OTs and ATPE are characterized by Fourier transform infrared spectroscopy, nuclear magnetic resonance hydrogen spectroscopy ( $\mathrm{H}^{1}$-NMR) and time of flight mass spectrometer. The characterization results show that the ATPE has a reasonable molecular weight, and the both hydroxyls of PEG-400 have been replaced by ethylenediamine. The inhibition property of ATPE, potassium chloride $(\mathrm{KCl})$, potassium formate $(\mathrm{KCOOH})$, methane-siliconic acid (FMS-1) and polymeric alcohol (JLX-B) are comparatively evaluated through shale rolling dispersion experiment, linear expansion experiment and laser particle size test. The results show that the ATPE has a better shale inhibition property than traditional inhibitors. The drilling fluid system performance evaluation results show that the system has good engineering application value with excellent rheological property, low fluid loss and good shale inhibition property, which guarantee the well-bore stability in shale gas drilling.
\end{abstract}

Keywords Amine terminated polyether inhibitor $\cdot$ Shale inhibition property $\cdot$ Shale recovery $\cdot$ Structural characterization - Well-bore stability

\section{Introduction}

As a type of clean energy, the shale gas can relieve the problem of energy stress, and it is significant for the stability of energy supply [1]. But in shale gas drilling, the stability of borehole is greatly influenced by the shale hydration, swelling and dispersion [2]. In order to solve these problems, it is imperative to develop a high efficiency shale inhibitor and a kind of water based drilling fluid system with high performance [3-5]. A series of amine type shale inhibitor using in water based drilling fluid system has been developed. In the design of the inhibitor molecular, the reasonable molecular weight, good water solubility and amine groups should be considered, which can inhibit the shale hydration [6]. The main synthetic methods of ATPE are catalytic reductive amination method and leaving group method $[7,8]$. Compared to catalytic reductive amination method, leaving group method has benefits from its mild reaction condition and simple synthesis equipment. The synthesis process does not require high temperature and pressure. So, the leaving group method is used to synthesize a new type of amine

$\triangle$ Xiaodong Bai, bxd888@vip.126.com; Vasileios Koutsos, vasileios.koutsos@ed.ac.uk|'School of Materials Science and Engineering, Southwest Petroleum University, Chengdu, China. ${ }^{2}$ School of Engineering, Institute for Materials and Processes, The University of Edinburgh, Edinburgh, UK.

SN Applied Sciences (2019) 1:94 | https://doi.org/10.1007/s42452-018-0112-x 
terminated polyether shale inhibitor with reasonable molecular weight. It is based on the molecule of PEG-400, and the two ends of the molecule are modified through two steps. Because of the amino structure at both ends of the molecular chain, the inhibitor can easily adsorb on the clay surface and enter the crystal layer. And then, the shale hydration and swelling are effectively inhibited [9-11]. The chemical reagents with low toxicity, low boiling point are used to improve the efficiency of purification and reduce environmental pollution. The structure and molecular weight of ATPE are characterized, and the shale inhibition property is evaluated. conversion rolling oven (type: GW300), and six-speed rotational viscometer (type: ZNN-D6B) were produced by Qingdao Tongchun Petroleum Instrument Co. (China).

\subsection{Synthesis of ATPE}

Under the condition of ice bath, PEG-400 and triethylamine were mixed together in a certain proportion, and then, $\mathrm{TsCl}$ was slowly dropped into the reaction flask at room temperature. The reaction process was tracked by thin-layer chromatography (TLC). The silica gel prefabricated board acted as TLC board. The developing agent was the mixture of dichloromethane and methanol with a mass ratio of 6:1. The reaction equation was as follows:

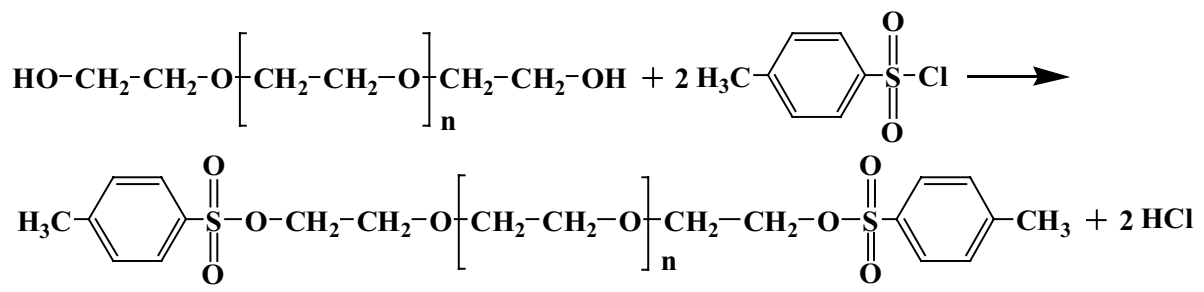

\section{Experimental}

\subsection{Materials and equipments}

PEG-400, P-toluenesulfonyl chloride ( $\mathrm{TsCl}$ ), ethylenediamine, triethylamine, potassium chloride and potassium formate were analytical reagents, which were provided by Chengdu Kelong Chemical Reagent Co. (China). Methane-siliconic acid (FMS-1), polymeric alcohol (JLX-B), coating agent (FA367), fluid loss additive (SMP-1), viscosifier (XC-HV) and lubricant (RH-220) were commercial products, which were purchased from Shandong Ziboruihou Commercial and Trade Co. (China). Nuclear magnetic resonance hydrogen spectroscopy (Bruker AVANCE III HD 400) was produced by Bruker Co. (Switzerland). Time of flight mass spectrometer (Agilent 6224) was obtained from Agilent Co. (America). Laser particle size analysis analyzer (MS 2000) was produced by Malvern Co. (England). Scanning electron microscope (ZEISS EVO MA15) was produced by Carl Zeiss Microscopic Image Co. Ltd (Germany). Shale expansion instrument (type: CPZ-2), mud medium pressure filtration apparatus (type: SD3), frequency
The precipitation in the liquid system was separated through vacuum filtration, and then, the distilled water and ethyle acetate were added to the liquid product. After standing and layering, the upper layer liquid was took out and added with sodium sulfate. The light yellow liquid was obtained after standing, filtration and vacuum distillation. Ethylether was added into the liquid, and then, the whole system was stratified after stirring and standing. The lower liquid was further purified through vacuum filtration. The intermediate product (PEG-OTs) was obtained.

Ethylenediamine and triethylamine were blended in certain proportion. PEG-OTs was previously dissolved in acetonitrile, and then, the PEG-OTs solution was dropped into the mixture of ethylenediamine and triethylamine at a certain temperature. The reaction process was tracked by thin-layer chromatography (TLC). The silica gel prefabricated board acted as TLC board. The developing agent was the mixture of dichloromethane and methanol with a mass ratio of 4:1. The reaction equation was as follows:

$\mathrm{H}_{3} \mathrm{C} \|_{\mathrm{O}}^{\mathrm{O}}-\mathrm{O}-\mathrm{CH}_{2}-\mathrm{CH}_{2}-\mathrm{O}-\mathrm{CH}_{2}-\mathrm{CH}_{2}-\mathrm{O}-\mathrm{CH}_{2}-\mathrm{CH}_{2}-\mathrm{O}-\mathrm{S}_{\mathrm{O}}^{\mathrm{O}} \longrightarrow \mathrm{CH}_{3}+2 \mathrm{H}_{2} \mathrm{~N}-\mathrm{CH}_{2}-\mathrm{CH}_{2}-\mathrm{NH}_{2} \rightarrow$

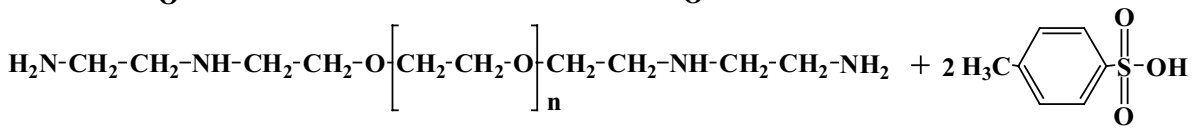


The organic solvent was removed from the primary product through vacuum filtration. Saturated sodium hydroxide solution and ethyl acetate were added in the system one after another. After layering, the upper layer liquid was took out to and added with sodium sulfate. The final light yellow liquid was ATPE, which was obtained after standing, filtration and vacuum distillation.

\subsection{Characterization of ATPE}

Fourier transform infrared spectrometer (NICOLET 6700) was used to characterize the molecular structures and the chemical compositions of PEG-400, PEG-OTs and ATPE respectively through the infrared absorption peaks of the functional groups. Before the test, potassium bromide was grinded and transferred into the mold to form a circular sheet. The samples were coated evenly on the potassium bromide sheet and tested with Fourier transform infrared spectrometer.

Nuclear magnetic resonance ( $\left.\mathrm{H}^{1}-\mathrm{NMR}\right)$ was used to analyze the chemical structure. $10 \mathrm{mg}$ pure sample was dissolved in $0.65 \mathrm{~mL}$ chloroform- $\mathrm{d}\left(\mathrm{CDCl}_{3}\right)$, and then, the solution was transferred to the nuclear magnetic sample tube. Nuclear magnetic resonance (Bruker AVANCE III HD 400) was used to record the proton absorption peaks of PEG-400, PEG-OTs and ATPE respectively.

Time of Flight Mass Sepectrometer (Agilent 6224) was used to detect the molecular weight distribution of PEG400, PEG-OTs and ATPE. The deionized water acted as solvent. The mobile phase included $30 \mathrm{wt} \%$ acetonitrile and 70 wt $\%$ deionized water. After injection, the PEG-400, PEG-OTs and ATPE were ionized through soft ionization technique, which was called electrospray ionization. The samples were scanned in positive mode respectively.

\subsection{Shale rolling dispersion experiment}

Several kinds of ATPE solution $(350 \mathrm{~mL})$ were prepared. $40.0 \mathrm{~g}$ Karamay Oilfield cuttings (6-10 mesh) were added into the solutions respectively. The suspension system was sealed in the aging cups and hot rolled at different temperatures $\left(80^{\circ} \mathrm{C}, 90^{\circ} \mathrm{C}, 100^{\circ} \mathrm{C}, 110^{\circ} \mathrm{C}\right.$ and $\left.120^{\circ} \mathrm{C}\right)$ for $16 \mathrm{~h}$ in the frequency conversion rolling oven (type: GW300). After hot rolling, the cuttings were sieved by 40 -mesh sieve after cooling, and the remaining cuttings were dried for $4 \mathrm{~h}$ at $105 \pm 3^{\circ} \mathrm{C}$. After weighting, the recovery rate was calculated as follows:

Recovery rate $=\frac{M}{40} \times 100(\%)$

$M(g)$ represents the weight of the remaining cuttings.

\subsection{Linear expansion experiment}

The bentonite $(10.0 \mathrm{~g})$ was dried for $4 \mathrm{~h}$ at $105 \pm 3^{\circ} \mathrm{C}$ before it was put into the test tube, which was filled with filter paper. The bentonite was compressed under the pressure of $10 \mathrm{MPa}$ for $5 \mathrm{~min}$, and then, a certain concentration of inhibitor solution was added into the test tube. The expansion rates of bentonite in each solution were tested and calculated:

Expansion rate $=\frac{\mathrm{H}_{8 \mathrm{~h}}}{\mathrm{H}_{0}} \times 100(\%)$

$\mathrm{H}_{8 \mathrm{~h}}(\mathrm{~mm})$ represents the expansion height of the bentonite core after $8 \mathrm{~h} ; \mathrm{H}_{0}(\mathrm{~mm})$ represents the original height of the bentonite core.

\subsection{Laser particle size test}

The certain mass fraction of ATPE, potassium chloride $(\mathrm{KCl})$, potassium formate $(\mathrm{KCOOH})$, methane-siliconic acid(FMS-1), and polymeric alcohol(JLX-B) were added to the pre-hydrated bentonite suspension ( $4.0 \mathrm{wt} \%$ ) respectively, and then, the suspensions were mixed under high speed stirring for $30 \mathrm{~min}$. The suspensions were for $24 \mathrm{~h}$ at room temperature, and then stirred for 10-15 min under high speed before they were added into the sample injector of the laser particle size analyzer (MS2000). When the particle concentration met the test requirements and the sufficient strength of the columnar data distribution of the shading degree was displayed, the small angle laser light scattering method was used to test the particle size distribution of the bentonite suspension.

\subsection{Microscopic morphology test}

Two bentonite solutions ( $4.0 \mathrm{wt} \%$ ) were prepared, one of which was added with ATPE (3 wt\%), the other one acted as blank sample. Dissolved and held for a period of time, took part of the solution and placed it in a steel sample box for freeze-drying, and then transferred the steel sample box to the testing room of ZEISS EVO MA15 electron microscope. At last, the micromorphology and shape of bentonite at different regions were respectively observed. The magnifications were 200 times.

\subsection{Performance evaluation of the ATPE drilling fluid}

The coating agent (FA367), fluid loss additive (SMP-1), viscosifier (XC-HV) and lubricant (RH-220) were optimized on the basis of ATPE. The water-based ATPE drilling fluid was composed of pre-hydrated bentonite suspension (4.0 wt\%), 
ATPE (4.0 wt\%), FA367 (0.3 wt\%), SMP-1 (1 wt\%), XC-HV (1 wt $\%), \mathrm{RH}-220$ (0.2 wt\%) and barite. The performance of ATPE drilling fluid was evaluated according to American Petroleum Institute (API) test programs. At first, the API filtration loss $\left(\mathrm{FL}_{\mathrm{API}}\right)$ of the mud was evaluated through the mud medium pressure filtration apparatus (SD3). Then, the aging tests was carried out by the frequency conversion rolling oven (type: GW300) through hot rolling at $120^{\circ} \mathrm{C}$ for $16 \mathrm{~h}$. During the filtration loss test, filter cake was formed on the filter paper, which can contribute to reduce the filtration loss. Apparently, if the filtration loss is small, the drilling fluid would have better performance. Finally, the rheological parameters of the drilling fluid were measured with the six-speed rotational viscometer (ZNN-D6B) at room temperature. Apparent viscosity (AV), plastic viscosity (PV), and yield point (YP) were evaluated through the numerical values when the rotational speed was $600 \mathrm{rpm}$ and $300 \mathrm{rpm}$. $\$ 600$ represents the numerical value shown on the indicator dial of the six-speed rotational viscometer when it was rotated with $600 \mathrm{rpm}$. $\$ 300$ represents the numerical value shown on the indicator dial of the six-speed rotational viscometer when it was rotated with $300 \mathrm{rpm}$. The computation formulas were as follows $[12,13]$ :

$\mathrm{AV}=1 / 2 \Phi 600(\mathrm{mPas})$

$\mathrm{PV}=\Phi 600-\Phi 300(\mathrm{mPa} \mathrm{s})$

$\mathrm{YP}=1 / 2(\Phi 300-\mathrm{PV})(\mathrm{Pa})$

\section{Results and discussion}

\subsection{Orthogonal experiment results and analysis}

The orthogonal $L_{16}\left(4^{4}\right)$ experiments are designed to optimize the synthesis conditions of amination reaction. The four main factors, including mole ratio of PEG-OTs/

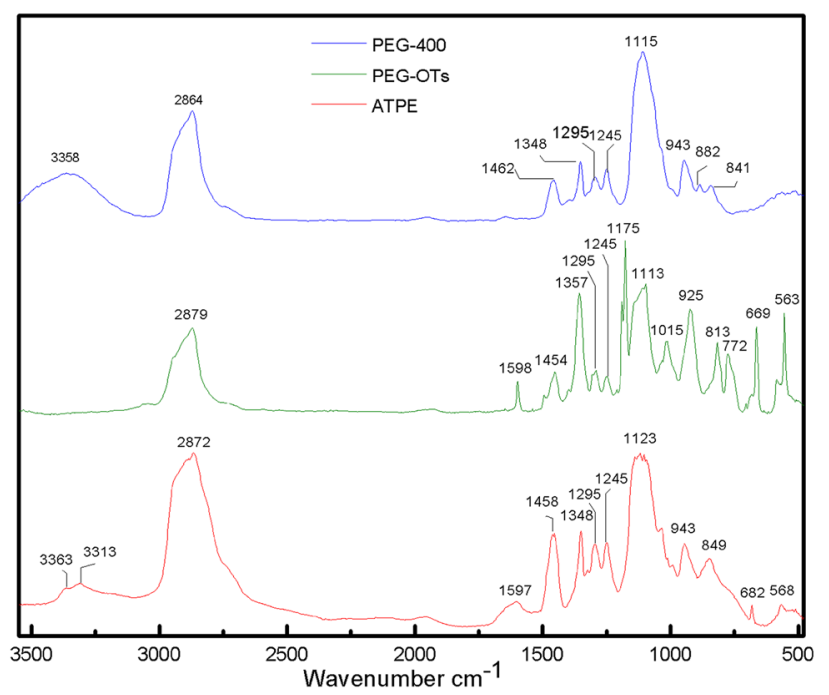

Fig. 1 FTIR of ATPE, PEG-OTs and PEG-400
Table 1 Orthogonal experimental results of amination reaction

\begin{tabular}{llllll}
\hline Sample & $\begin{array}{l}\mathrm{n}(\mathrm{PEG}-\mathrm{OTs}): \\
\mathrm{n}(\text { ethylenediamine) }[\mathrm{A}]\end{array}$ & $\begin{array}{l}\mathrm{n} \text { (PEG-OTs): } \\
\mathrm{n} \text { (triethylamine) }[\mathrm{B}]\end{array}$ & $\begin{array}{l}\text { Reaction } \\
\text { temperature/ }{ }^{\circ} \mathrm{C}[\mathrm{C}]\end{array}$ & $\begin{array}{l}\text { Reaction } \\
\text { time/h }[\mathrm{D}]\end{array}$ & Yield/\% \\
\hline 1 & $1: 2.4$ & $1: 2.5$ & 50 & 2.0 & 73.65 \\
2 & $1: 2.4$ & $1: 3.0$ & 60 & 2.5 & 82.33 \\
3 & $1: 2.4$ & $1: 3.5$ & 70 & 3.0 & 84.64 \\
4 & $1: 2.4$ & $1: 4.0$ & 80 & 3.5 & 84.57 \\
5 & $1: 2.8$ & $1: 2.5$ & 60 & 3.0 & 87.76 \\
6 & $1: 2.8$ & $1: 3.0$ & 50 & 3.5 & 85.42 \\
7 & $1: 2.8$ & $1: 3.5$ & 80 & 2.0 & 79.78 \\
8 & $1: 2.8$ & $1: 4.0$ & 70 & 2.5 & 88.54 \\
9 & $1: 3.2$ & $1: 2.5$ & 70 & 3.5 & 86.67 \\
10 & $1: 3.2$ & $1: 3.0$ & 80 & 3.0 & 86.31 \\
11 & $1: 3.2$ & $1: 3.5$ & 50 & 2.5 & 88.69 \\
12 & $1: 3.2$ & $1: 4.0$ & 60 & 2.0 & 87.33 \\
13 & $1: 3.6$ & $1: 2.5$ & 80 & 2.5 & 85.03 \\
14 & $1: 3.6$ & $1: 3.0$ & 70 & 2.0 & 86.79 \\
15 & $1: 3.6$ & $1: 3.5$ & 60 & 3.5 & 91.04 \\
16 & $1: 3.6$ & $1: 4.0$ & 50 & 3.0 & 87.07 \\
Kj1 & 81.297 & 83.278 & 83.707 & 81.888 & \\
Kj2 & 85.375 & 85.213 & 87.115 & 86.148 & \\
Kj3 & 87.250 & 86.038 & 86.660 & 86.445 & \\
Kj4 & 87.483 & 86.877 & 83.922 & 86.925 & \\
Rj & 6.186 & 3.599 & 3.408 & 5.037 & \\
\hline
\end{tabular}


ethylenediamine, mole ratio of PET-OTs/triethylamine, reaction temperature and reaction time are selected to research the synthesis conditions as listed in Table 1.

Setting the yield of ATPE as index, the order of the influence on the yield is $n$ (PEG-OTs): $n$ (ethylenediamine) $>$ reaction time $>\mathrm{n}$ (PEG-OTs): $\mathrm{n}$ (triethylamine) $>$ temperature. According to the orthogonal experiment results, the order of main factors is ADBC. The optimal level is A4D4B4C2. The optimized synthesis conditions are as follows: mole ratio of PEG-OTs/ethylenediamine is $1 / 3.6$, the reaction time is $3.5 \mathrm{~h}$, the mole ratio of PEG-OTs/triethylamine is $1 / 4$ and reaction temperature is $60^{\circ} \mathrm{C}$.

\subsection{FTIR analysis of ATPE}

The spectrum shows the infrared characteristic absorption peaks of PEG-400, PEG-OTs and ATPE, which are shown in Fig. 1.

In the spectrum of PEG-400, the absorption peaks at $3358 \mathrm{~cm}^{-1}$ and $2864 \mathrm{~cm}^{-1}$ respectively correspond to the stretching vibration of $-\mathrm{OH}$ and $-\mathrm{CH}_{2}-$. The absorption peak at $1115 \mathrm{~cm}^{-1}$ corresponds to the anti-symmetric stretching vibration of $\mathrm{C}-\mathrm{O}-\mathrm{C}$. The Infrared characterization of the middle production PEG- OTs shows that the main chain structure of PEG-400 is unchanged. The disappeared absorption peak at $3358 \mathrm{~cm}^{-1}$ indicates that the terminal hydroxyl of PEG-400 has been replaced. The absorption peaks at $1357 \mathrm{~cm}^{-1}$ and $1175 \mathrm{~cm}^{-1}$ respectively correspond to the anti-symmetric stretching vibration and symmetric stretching vibration of $\mathrm{O}=\mathrm{S}=\mathrm{O}$. The Infrared characterization of ATPE indicates that $3363 \mathrm{~cm}^{-1}$,

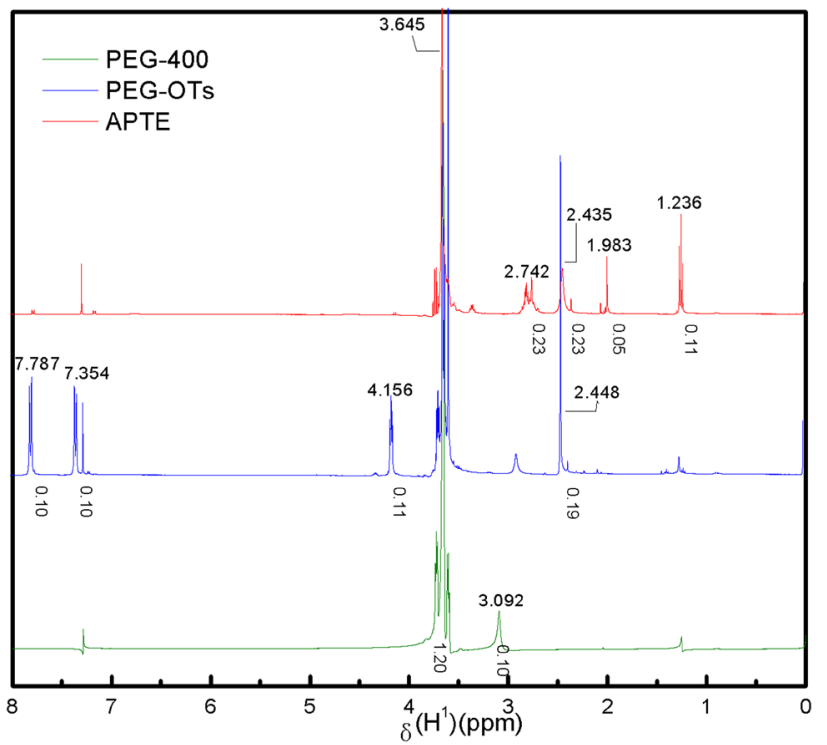

Fig. $2 H^{1}$-NMR of ATPE, PEG-OTs and PEG-400
$3313 \mathrm{~cm}^{-1}$ and $1597 \mathrm{~cm}^{-1}$ respectively correspond to the anti-symmetric stretching, symmetric stretching and variable angle stretching vibration peaks of $-\mathrm{NH}_{2}$. The symmetric stretching vibration peak of $\mathrm{O}=\mathrm{S}=\mathrm{O}$ at $1175 \mathrm{~cm}^{-1}$ disappears.

\section{3 $H^{1}$-NMR analysis of ATPE}

The nuclear magnetic resonance hydrogen spectrum ( $\mathrm{H}^{1}$-NMR) of PEG-400, PEG-OTs and ATPE are shown in Fig. 2.

In the $\mathrm{H}^{1}$-NMR spectrum of PEG-400, the absorption peaks at $3.662 \mathrm{ppm}$ and $3.092 \mathrm{ppm}$ respectively correspond to the proton peak of $-\mathrm{O}-\mathrm{CH}_{2}-\mathrm{CH}_{2}-\mathrm{O}$ - and $-\mathrm{OH}$. The peak area ratio is 12:1. In the $\mathrm{H}^{1}$-NMR spectrum of PEG-OTs, the absorption peak at 2.448 ppm corresponds to the proton peak of $-\mathrm{CH}_{3}$ on the benzene ring. The absorption peak at $4.156 \mathrm{ppm}$ corresponds to the proton peak of $-\mathrm{CH}_{2}-$ which links with sulfonic acid ester. The absorption peak at $7.787 \mathrm{ppm}$ corresponds to the proton peak of $C$ which is on the benzene ring at 3-positon. The absorption peak at $7.354 \mathrm{ppm}$ corresponds to the proton peak of $C$ which is on the benzene ring at 4-positon. The peak area ratio of $2.448 \mathrm{ppm}$ and $4.156 \mathrm{ppm}$ is $2: 3$, which indicates that the intermediate product is double substituted sulfonic acid ester. In the $\mathrm{H}^{1}$-NMR spectrum of ATPE, the absorption peak at $3.654 \mathrm{ppm}$ corresponds to the proton peak of $-\mathrm{O}-\mathrm{CH}_{2}-\mathrm{CH}_{2}-\mathrm{O}-$. The absorption peaks at $2.742 \mathrm{ppm}$ and $2.435 \mathrm{ppm}$ respectively correspond to the proton peak of $-\mathrm{NH}-\mathrm{CH}_{2}-\mathrm{CH}_{2}-\mathrm{NH}_{2}$ and $-\mathrm{NH}-\mathrm{CH}_{2}-\mathrm{CH}_{2}-\mathrm{O}-$. The absorption peak at 1.983 ppm corresponds to the proton peak of $-\mathrm{NH}-$. The absorption peak at $1.236 \mathrm{ppm}$ corresponds to the proton peak of $-\mathrm{NH}_{2}$. The integral area ration of $-\mathrm{NH}-$ and $-\mathrm{NH}_{2}$ is $1: 2$.

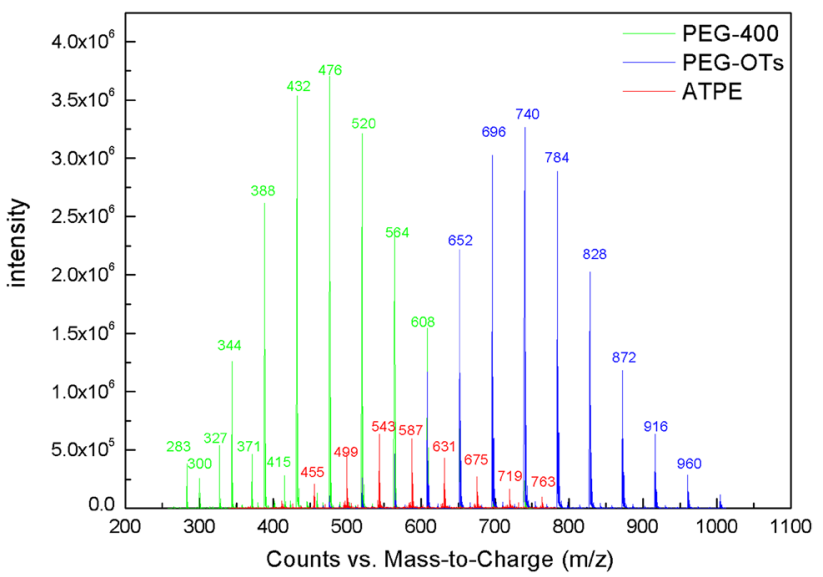

Fig. 3 Mass spectrogram of PEG-400, PEG-OTs and ATPE 
Table 2 Mass spectrometry analysis results of PEG-400, PEG-OTs and ATPE

\begin{tabular}{|c|c|c|c|c|c|c|c|c|c|}
\hline \multirow{2}{*}{$\begin{array}{l}\text { Numbers of repeat- } \\
\text { ing structure unit/n }\end{array}$} & \multicolumn{3}{|c|}{ Theoretical molecular weight } & \multicolumn{3}{|c|}{ Mass-to-charge ratio (m/z) } & \multicolumn{3}{|c|}{ The measured molecular weight/u } \\
\hline & $M(P E G-400)$ & M(PEG-OTs) & $M(A T P E)$ & $\begin{array}{l}\text { PEG-400/ } \\
\left(\mathrm{M}+\mathrm{NH}_{4}\right)^{+}\end{array}$ & $\begin{array}{l}\text { PEG-OTs/ } \\
\left(\mathrm{M}+\mathrm{NH}_{4}\right)^{+}\end{array}$ & $\begin{array}{l}\text { ATPE/ } \\
\left(\mathrm{M}+\mathrm{NH}_{4}\right)^{+}\end{array}$ & $M(P E G-400)$ & M(PEG-OTs) & M(ATPE) \\
\hline 7 & 370 & 678 & 454 & 388 & 696 & 455 & 370 & 678 & 454 \\
\hline 8 & 414 & 722 & 498 & 432 & 740 & 499 & 414 & 722 & 498 \\
\hline 9 & 458 & 766 & 542 & 476 & 784 & 543 & 458 & 766 & 542 \\
\hline 10 & 502 & 810 & 586 & 520 & 828 & 587 & 502 & 810 & 586 \\
\hline 11 & 546 & 854 & 630 & 564 & 872 & 631 & 546 & 854 & 630 \\
\hline 12 & 590 & 898 & 674 & 608 & 916 & 675 & 590 & 898 & 674 \\
\hline 13 & 634 & 942 & 718 & 652 & 960 & 719 & 634 & 942 & 718 \\
\hline
\end{tabular}

Table 3 Shale rolling dispersion experiment results

\begin{tabular}{lllllllll}
\hline $\mathrm{T}\left({ }^{\circ} \mathrm{C}\right)$ & \multicolumn{2}{l}{ Recovery rate $(16 \mathrm{~h}) / \%$} & & & \\
\cline { 2 - 8 } & Distilled water & 1 wt\% ATPE & 2 wt\% ATPE & 3 wt\% ATPE & 7 wt\% KCl & 7 wt\% KCOOH & 3 wt\% FMS-1 & 3 wt\% JLX-B \\
\hline 80 & 12.9 & 61.35 & 65.13 & 71.33 & 36.48 & 38.93 & 41.6 & 35.23 \\
90 & 12.8 & 61.05 & 64.92 & 71.2 & 36.26 & 38.74 & 41.42 & 35.07 \\
100 & 12.65 & 60.73 & 64.69 & 70.5 & 36.13 & 38.66 & 41.29 & 34.93 \\
110 & 12.4 & 60.51 & 64.3 & 70.36 & 36.02 & 38.52 & 41.07 & 34.81 \\
120 & 12.1 & 60.05 & 64.24 & 70.19 & 35.91 & 38.40 & 40.89 & 34.68 \\
\hline
\end{tabular}

\subsection{Time of flight mass spectrometer analysis}

The mass distribution of the molecular ion of test samples is shown in Fig. 3. The analysis of the results is shown in Table 2.

As the results shown from Fig. 3, the difference between the signals of mass-to-charge ratio in each group of peaks is 44 , which corresponds to the molecular repetitive structure unit of $-\mathrm{CH}_{2}-\mathrm{CH}_{2}-\mathrm{O}-$. In Fig. 3, the $\mathrm{m} / \mathrm{z}$ signals 283 , $327,371,415,459$ are the $(\mathrm{M}+\mathrm{H})^{+}$ion signals of PEG-400 when $n$ is 5-9, which supports the accuracy of the molecular weight of PEG-400. As shown in Table 2, the characterization testing values of PEG-400, PEG-OTS and ATPE match with the theoretical calculations, which further prove that the intermediate product and the target product are synthesized successfully. The molecular weight distribution of ATPE is mainly from 454 to 718 . The molecular weight is reasonable and met with the design requirements. Studies have shown that low molecular weight amines can be inserted into the clay crystal layer [14, 15]. ATPE has reasonable molecular weight, which makes it can enter the clay mineral crystal layer. And then, the adjacent crystal layer spacing is compressed and the water molecules are pushed out. As a result, the clay is not easy to hydrate and disperse.

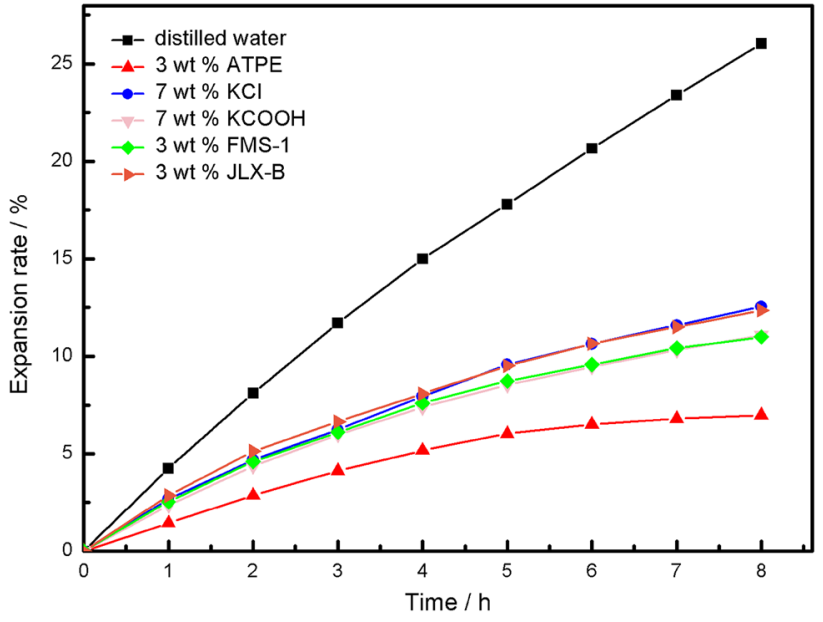

Fig. 4 Linear expansion experiment results

\subsection{Analysis of shale rolling dispersion}

Shale rolling dispersion experiment is used to evaluate the shale inhibitive effect of ATPE under high temperature for a long aging time. The experimental results are shown in Table 3.

As is shown in Table 3, the shale recovery rate in the $3 \mathrm{wt} \%$ ATPE solution is the highest under different temperature. With the increasing of temperature, the recovery rate of shale gradually decreases. The main reason is that 
the increasing of temperature promotes the hydration and dispersion of shale. On the other hand, the shale inhibitive effect is better than other traditional inhibitors such as $\mathrm{KCl}, \mathrm{KCOOH}, \mathrm{FMS}-1$ and JLX-B. The experiments indicate that ATPE has a strong ability to inhibit the dispersion and hydration of shale under high temperature for a long aging time. Because ATPE is a kind of nonionic shale inhibitor, the mutual repulsion of the negative charges on the shale surface is weakened by the adsorption of ATPE. As a result, the clay is not easy to hydrate and disperse.

\subsection{Linear expansion experiment analysis}

Linear expansion experiment is used to evaluate the shale inhibitive effect of ATPE under normal pressure and temperature. The results are shown in Fig. 4.

As is shown in Fig. 4, the expansion rate of bentonite core has a minimum value in the 3 wt\% ATPE solution, which is only $6.99 \%$. Compared with other traditional inhibitors, such as $\mathrm{KCl}, \mathrm{KCOOH}$, FMS-1 and JLX-B, The shale inhibitive effect of ATPE is better. The experiments indicate that ATPE has a strong ability to inhibit the dispersion and hydration of shale under normal pressure and temperature. ATPE molecules adsorbed on the clay surface, the hydrophobic chain in ATPE results in the hydrophobic clay surface, while the hydrophilic property of the

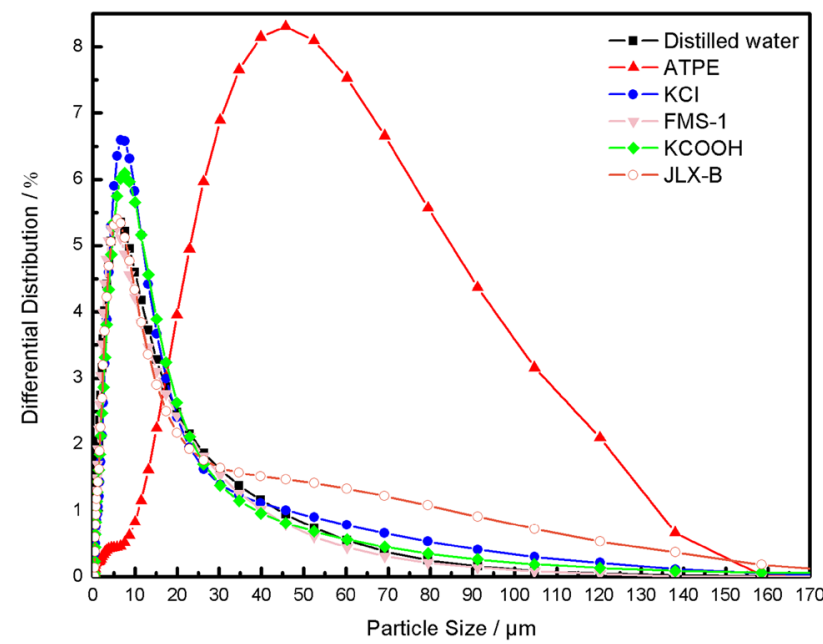

Fig. 5 Particle size distribution of bentonite suspensions with different inhibitors clay surface is weakened. Water molecules are prevented to enter into the clay crystal layers, as a result, the clay is not easy to hydrate and disperse. Ma [16] synthesized an inhibitor DAM by diallyl dimethyl ammonium chloride (DMDACC), methyl allyl alcohol (MP), 2-acrylamido-2-methyl propane sulfonic acid (AMPS). When the DAM dosage was $3 \mathrm{wt} \%$, the linear expansion rate was $16 \%$. Zhong [17] used 4,4'-methylenebis-cyclohexanamine (MBCA) as shale inhibitor and added to bentonite solution, the linear expansion rate was $10 \%$ after $5 \mathrm{~h}$. An [18] added $4 \mathrm{wt} \%$ polyethyleneimine (PEI) to montmorillonite solution, and the linear expansion decreases by $73 \%$ after $6 \mathrm{~h}$. Comparatively, the linear expansion decreases by $75 \%$ after adding $3 \mathrm{wt} \%$ APTE for $6 \mathrm{~h}$ in this paper.

\subsection{Particle size and distribution analysis}

The bentonite suspensions with different inhibitors are tested by laser particle size analyzer (MS 2000). The results are shown in Fig. 5 and Table 4.

As the results shown above, the average diameter of the clay particles in $3 \mathrm{wt} \%$ ATPE solution increases from 5.479 to $37.533 \mu \mathrm{m}$ compared with distilled water. The controlling effects of the other traditional inhibitors on the average particle size of bentonite are not obvious. The order of average particle size in different suspensions is ATPE $>\mathrm{KCl}>\mathrm{KCOOH}>\mathrm{JLX}-\mathrm{B}>$ distilled water $>$ FMS -1 , which proves that ATPE has a strong inhibition ability to clay dispersion.

\subsection{Micromorphology analysis of bentonite particles}

The SEM is used to observe the micromorphology of bentonite particles in different solutions, and the images are shown in Fig. 6.

As shown in Fig. 6, the surface hydration of bentonite particles in blank sample is more serious, which can be proved by the porous structure on the bentonite surfaces. The bentonite particles are smaller and more dispersive. When ATPE is added, the surface hydration of bentonite particles is greatly weakened and the particles are more aggregated. The main reason is that ATPE is a kind of nonionic shale inhibitor, which can adsorb on the bentonite surface and shield the original negative charge on the
Table 4 Laser particle size experiment results

\begin{tabular}{lllllll}
\hline Samples & Distilled water & 3 wt\% ATPE & 7 wt\% KCl & 7 wt\% KCOOH & 3 wt\% FMS-1 & 3 wt\% JLX-B \\
\hline $\mathrm{d}(0.5) / \mu \mathrm{m}$ & 5.479 & 37.533 & 6.565 & 6.394 & 5.024 & 6.071 \\
$\begin{array}{l}\text { Specific } \\
\begin{array}{l}\text { surface area/ } \\
\left(\mathrm{m}^{2} \mathrm{~g}^{-1}\right)\end{array}\end{array}$ & 1.880 & 0.391 & 1.460 & 1.620 & 1.980 & 1.700 \\
\hline
\end{tabular}




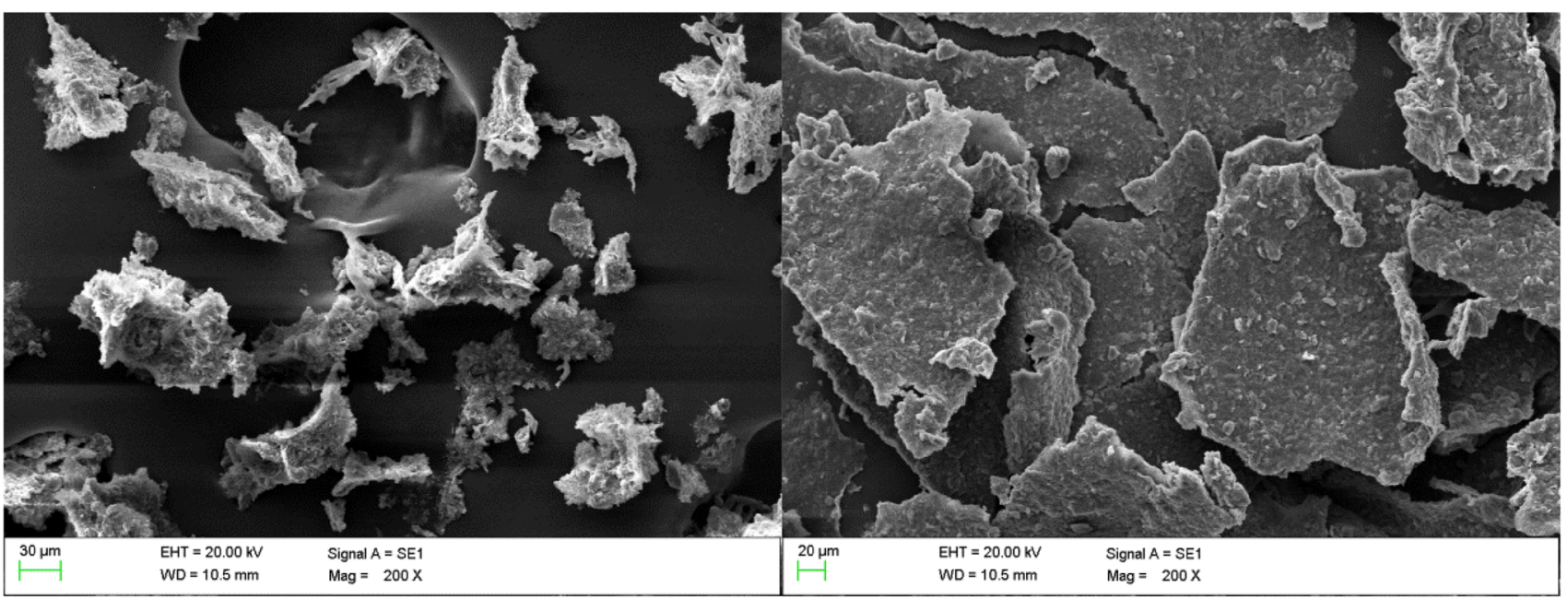

(a) SEM of bentonite blank sample

(b) SEM of bentonite with $3 \mathrm{wt} \%$ ATPE

Fig. 6 SEM of bentonite particles in different solutions

Table 5 Performance evaluation results of ATPE drilling fluid

\begin{tabular}{|c|c|c|c|c|c|c|c|}
\hline Sample & Conditions & $\begin{array}{l}\text { Sample recovery } \\
\text { weight }(16 \mathrm{~h}) / \mathrm{g}\end{array}$ & $\begin{array}{l}\text { Recovery rate } \\
(16 \mathrm{~h}) / \%\end{array}$ & $\mathrm{AV} / \mathrm{mPa} \mathrm{s}$ & $\mathrm{PV} / \mathrm{mPa} \mathrm{s}$ & $\mathrm{YP} / \mathrm{Pa}$ & $\mathrm{FL}_{\mathrm{API}} / \mathrm{mL}$ \\
\hline \multirow[t]{2}{*}{ Optimizing composition } & Before aging & 37.00 & 92.50 & 58 & 39.5 & 18.5 & 4.3 \\
\hline & After aging & & & 56 & 38 & 18 & 5.1 \\
\hline
\end{tabular}

surface. As a result, the electrostatic repulsion between the bentonite particles is decreased, and the bentonite particles are more easily aggregated. Secondly, as a small molecular polymer, ATPE can insert into the double-layer structure of bentonite and squeeze out the water molecules. At last, the hydration and expansion of bentonite are inhibited.

\subsection{Performance of ATPE drilling fluid}

The water-based ATPE drilling fluid is composed of prehydrated bentonite suspension (4.0 wt\%), ATPE (4.0 wt\%), FA367 (0.3 wt\%), SMP-1 (1 wt\%), XC-HV (1 wt\%), RH-220 (0.2 wt\%) and barite. The performance of ATPE drilling fluid was evaluated according to American Petroleum Institute (API) test programs. The shale recovery, the rheological parameters and API filtration are tested respectively. The results are shown in Table 5.

As is shown in Table 5, the shale recovery rate reaches $92.5 \%$ due to the synergistic effect of the coating agent and the inhibitor, which demonstrates the excellent shale inhibition property of ATPE drilling fluid. In addition, the AV, PV, YP and $\mathrm{FL}_{\mathrm{API}}$ of ATPE drilling fluid have little change. The $\mathrm{FL}_{\mathrm{API}}$ is less than $5.1 \mathrm{~mL}$ for the ATPE drilling fluid after the thermal aging at $120^{\circ} \mathrm{C}$ for $16 \mathrm{~h}$.

\section{Conclusions}

A new type of amine terminated polyther (ATPE) shale inhibitor used in water-based drilling fluid is synthesized through the method of leaving group, and the optimal polymerization conditions are obtained: the mole ratio of polyethylene glycol tosylate (PEG-OTs)/ethylenediamine is $1 / 3.6$, mole ratio of PEG-OTs/triethylamine (acid binding agent) is $1 / 4$, reaction time is $3.5 \mathrm{~h}$ and reaction temperature is $60^{\circ} \mathrm{C}$. The structure of ATPE is characterized by FTIR, $\mathrm{H}^{1}$-NMR and TOFMS. The results show that ATPE contains all the designed functional groups and reasonable molecular weight. The shale recovery rate of ATPE for $16 \mathrm{~h}$ reaches $71.33 \%$ and the $8 \mathrm{~h}$ linear expansion rate is only $6.99 \%$. ATPE makes the specific surface area of prehydrated bentonite particles reach the relatively minimum value, which is $0.391 \mathrm{~m}^{2} / \mathrm{g}$. The average diameter of the clay particles in 3 wt $\%$ ATPE solution increases from 5.479 to $37.533 \mu \mathrm{m}$ compared with distilled water. All the results prove that the inhibitive effect of ATPE is superior to other traditional inhibitors. ATPE drilling fluid has strong shale inhibition property, good rheological property and filtration property. 
Acknowledgements This article was supported by the Gas and Oil Fields Materials Key Laboratory of the Colleges of Sichuan Province (Serial Number: X151516KCL36). It was also supported by the National Natural Science Foundation of China (NSFC). The name of the research item is "Dispersion process and action principle with multicomponent of nanoparticles in drilling fluid," and the item serial number is 51104122 .

\section{Compliance with ethical standards}

Conflict of interest On behalf of all authors, the corresponding author states that there is no conflict of interest.

\section{References}

1. Zou CH, Zhu RK, Wu ST (2012) Types, characteristics, genesis and prospects of conventional and unconventional hydrocarbon accumulations: taking tight oil and tight gas in China as an instance. Acta Pet Sin 33(2):173-187

2. Browning WC (1964) The hydroxyl factor in shale control. JPT 16(10):1177-1186

3. Qiu ZS, Zhong HY, Huang WA (2011) Properties and mechanism of a new polyamine shale inhibitor. Acta Pet Sin 32(4):678-682

4. Patel A, Stamatakis E, Davis E (2001) Shale hydration inhibition agent and method of use. US Patent, 6247543

5. Bishop BS, Robert DG (1985) Interactions of secondary amines with bentonite clay and humic materials in dilute aqueous systems. Environ Int 11(6):499-504

6. Qu YZ (2010) Study on a new amine shale inhibitor (I): the molecular design and synthesis. Drill Fluid Complet Fluid 27(1):1-3

7. Veerapandiana S, Sultan NA (2015) Amine-and blocked isocyanate-terminated polyurethane dendrimers: integrated synthesis, photophysical properties and application in a heat curable system. RSC Adv 5(5):3799-3806

8. Sun S, Quan ZJ, Wang XC (2015) Selective reduction of nitrocompounds to primary amines by nickel-catalyzed hydrosilylative reduction. RSC Adv 5(103):84574-84577

9. Bongwoo H, Kookheon C (2005) Conformational behavior of dodecyldiamine inside the confined space of montmorillonites. Langmuir 21(18):8471-8477

10. Zhong HY, Qiu ZS, Huang WA et al (2015) Synergistic stabilization of shale by a mixture of polyamidoamine dendrimers modified bentonite with various generations in water-based drilling fluid. Appl Clay Sci 114:359-369

11. Xian Y, Jiang GC, Li YY, Yang LL, Zhang XM (2015) Biodegradable oligo (poly-L-lysine) as a high-performance hydration inhibitor for shale. RSC Adv 5(103):84947-84958

12. Yan JN (2006) Drilling fluids technology. China University of Petroleum Press, Shangdong

13. Bai XD, Yang Y, Xiao DY, Pu XL (2015) Synthesis, characterization, and performance evaluation of the AM/AMPS/DMDAAC/ SSS quadripolymer as a fluid loss additive for water-based drilling fluid. J Appl Polym Sci 132(14):27-34

14. Lee L, Patel A. D, Stamatakis E (2001) Glycol based drilling fluid. US Patent, 0155157

15. Stamatakis E (2007) High performance water based drilling mud and method of Use. US Patent 7250390

16. Ma F, Pu X, Wang B (2017) Preparation and evaluation of polyampholyte inhibitor DAM. RSC Adv 7(78):49320-49328

17. Zhong HY, Qiu ZS, Tang ZC (2016) Study of 4, 4'-methylenebiscyclohexanamine as a high temperature-resistant shale inhibitor. J Mater Sci 51(16):7585-7597

18. An YX, Yu PZ (2016) A strong inhibition of polyethyleneimine as shale inhibitor in drilling fluid. J Pet Sci Eng 161:1-8 